Horizons philosophiques

\title{
Martin Eden ou la quête du bonheur en Amérique
}

\section{Michèle Émond}

Volume 14, numéro 1, automne 2003

$\mathrm{Au}$ risque du bonheur

URI : https://id.erudit.org/iderudit/801252ar

DOI : https://doi.org/10.7202/801252ar

Aller au sommaire du numéro

\section{Éditeur(s)}

Collège Édouard-Montpetit

\section{ISSN}

1181-9227 (imprimé)

1920-2954 (numérique)

Découvrir la revue

\section{Citer cet article}

Émond, M. (2003). Martin Eden ou la quête du bonheur en Amérique. Horizons philosophiques, 14(1), 77-92. https://doi.org/10.7202/801252ar d'utilisation que vous pouvez consulter en ligne.

https://apropos.erudit.org/fr/usagers/politique-dutilisation/ 


\section{MARTIN EDEN OU LA QUÊTE DU BONHEUR EN AMÉRIQUE}

"Une vie, c'est l'histoire de cette vie, en quête de narration"1". Paul Ricœur

"I'd rather be ashes than dust."

Jack London

L'American dream a porté plusieurs noms dans l'histoire idéologique américaine. Lors de la Déclaration d'Indépendance, les révolutionnaires américains s'étaient inspirés de la théorie du père du libéralisme politique John Locke qui établissait que les hommes étaient dotés de droits naturels fondamentaux, à savoir la vie, la liberté et le droit de propriété. Les Américains ont repris cette idée en remplaçant le droit de propriété par la recherche du bonheur. L'ironie de l'histoire est que l'idéologie libérale classique considérait le droit de propriété comme la quintessence du bonheur'! Aujourd'hui, le rêve américain reste surtout l'expression du rêve d'accession à la richesse et au monde de la consommation qui fait le bonheur des classes moyennes et l'envie des habitants des pays du Sud, mais il a été successivement le rêve d'espace et de vie saine des pionniers de la frontière, puis le rêve démocratique de l'égalité des droits et des chances d'accéder à l'éducation et à la direction politique du pays. II a aussi été le rêve d'aventuriers de la route et de l'esprit en quête d'une autre Amérique : hobos, tramps, chercheurs d'or du début du $20 \mathrm{e}$ siècle; aventuriers des trottoirs des villes aujourd'hui : hustlers et amants de la dive bouteille et autres paradis artificiels. L'histoire de la littérature américaine pourrait s'écrire en nommant successivement les chantres de ce rêve et de ses désillusions : Mark Twain, Thoreau, London, Kerouac, Dos Passos, Henry Miller en sont quelques-uns.

Le récit de la quête de l'un de ces aventuriers par Jack London peut servir de paradigme à l'élaboration de ce rêve et comme critique exemplaire de la voie individualiste d'accès au bonheur au début du vingtième siècle. Martin Eden ${ }^{3}$ est le titre de ce roman partiellement autobiographique de Jack London publié en 1909. II raconte la longue ascension sociale de Martin Eden, jeune matelot de vingt ans qui débarque à San Francisco, fait la connaissance de Ruth Morse, jeune 
fille de la bonne bourgeoisie et tente de la conquérir en devenant écrivain et célèbre. Ce roman a connu un succès important dès le début et pas tout à fait pour les bonnes raisons. L'exaltation de la volonté de puissance y triomphe comme source ultime du bonheur alors que la fin du récit livre plus sûrement le sens moral souhaité par l'auteur. Échec d'une quête philosophique, récit d'une ascension sociale accompagnée de désillusions amoureuses et d'une impossible rencontre entre deux mondes sociaux, le roman se termine avec le suicide de Martin Eden, se laissant couler dans l'océan. Les idées philosophiques qui avaient cours dans les cercles intellectuels progressistes fréquentés par Jack London, soit le socialisme et le darwinisme social, couplées à la critique de la théorie du surhomme de Nietzsche vont servir de support pour illustrer les tensions sociales et psychiques qui jalonnent la quête du succès de London et celle du bonheur de Martin Eden. La question sera donc de savoir si la fin du roman, soit le suicide de Martin Eden doit faire conclure à l'échec de cette voie vers le bonheur.

\section{Fiction, récit et réalité}

Il est tentant de tisser plusieurs liens entre la biographie de Jack London et l'itinéraire personnel qu'il tracera pour son héros. Une idée tenace fait de ce roman de London une biographie à peine déguisée et les critiques se demandaient pourquoi l'auteur s'en défendait, tant il $y$ a de points communs entre les "personnages" principaux de la vie de London et ceux du roman : les héros sont de jeunes écrivains; ils ont sensiblement la même enfance, les mêmes expériences de vie; leurs amoureuses et leurs amis ont les mêmes traits et ils vivent à la même époque. C'est à dessein que le terme de personnage est choisi pour situer l'écrivain, puisque la fabrication d'une légende autour de son nom s'est construite dès le début de sa carrière et London luimême n'est pas étranger à la mise en récit romanesque de plusieurs aspects de sa vie. Même si le genre littéraire choisi par l'auteur - le récit d'aventures - le réalisme de son style et l'immense vitalité, presque archétypale de ses personnages n'avaient pas induit une vie hors du commun de son auteur, la liste des expériences de vie de London en fait un personnage aux visages multiples et aux identités sociales complexes. Le souvenir de ses conférences socialistes enflammées a aussi nourri l'image d'un individu défiant l'ordre établi, ce qui lui procure une place de choix dans le palmarès des individualistes rebelles, figures mythiques de l'idéologie romantique 
américaine 4 . La légende s'est accentuée avec sa mort précoce et mystérieuse à 40 ans en 1916. London aurait mis fin à ses jours en avalant des somnifères, selon la légende, ou serait mort des suites d'une maladie des reins, selon des sources plus documentées. L'embargo assez longuement maintenu sur ses papiers personnels par sa famille a fait le reste ${ }^{5}$. Quand la fiction marque un destin, il est tentant d'adapter cette expression célèbre en littérature : "Jack London n'était pas Martin Eden mais il le devint6».

Pourtant, qui dit récit dit aussi choix, point de vue, liberté avec les faits et les attitudes face à ces faits, ce qui est loin d'une démarche biographique ou autobiographique qui suppose que l'auteur ou son historien accepte de creuser ses intentions, d'expliquer ses choix et de se soumettre au jugement des faits et des autres. Raconter sa vie et écrire un roman, cela n'est donc pas la même chose. D'autre part, et comme le souligne Paul Ricoeur, "le récit fait partie de la vie avant de s'exiler de la vie dans l'écriture; il fait retour à la vie selon (les) voies multiples et au prix (de) tensions inexpugnables ${ }^{7}$ '. Toute vie, tout homme est en quête de narration. Faire de sa vie une histoire cohérente, qui a un sens et qui peut être racontée, acceptée par les autres et par soi, est au cœur de la quête philosophique moderne. La recherche du bonheur, du bien, suppose ainsi qu'un être puisse construire un récit où les différents événements puissent s'accorder, s'enchaîner, s'expliquer en accordant le statut de sujet à celui qui produit cette histoire. Agent de son histoire, le sujet est en quête d'un récit qui sera le sien. En écrivant l'histoire de la vie de Martin Eden, London esquissait aussi un récit possible de sa propre vie.

Que veut donc faire London en commençant ce roman autour d'un jeune homme qui lui ressemblait il y a dix ans, au début de sa vingtaine, quand il n'avait pas encore connu le succès? Se servir de son expérience pour assurer la crédibilité de l'histoire, cela allait de soi, pour London. II écrit Martin Eden en 1907-1908, alors qu'il vogue dans le Pacifique sur le Snark en compagnie de sa femme Charmian. L'écrivain d'expérience qu'il est devenu sait se détacher de la tentation de traduire directement ses expériences et ses sentiments et choisir les éléments de sa vie qui pourront captiver son auditoire et faire avancer le récit. London est aussi chroniqueur social et moraliste. Journaliste, parce que ses descriptions sont autant d'observations minutieuses et dépassionnées des êtres et des milieux. S'il s'amuse parfois à noircir des personnages réels et à donner libre cours à ses préjugés, il sait aussi toucher du doigt les 
infimes subtilités des différences de classe. Moraliste, parce qu'il livre un message politique à travers l'évocation d'un drame personnel qui est aussi lillustration de la lutte pour la vie au cœur de la lutte des classes, pour le socialiste London. Aussi, quand il se défend d'avoir écrit ici sa biographie, il dit surtout qu'il n'a pas cherché à clarifier ses propres rapports au succès, à la gloire. Cela expliquerait qu'il choisit de donner une philosophie extrémiste à son personnage, sorte d'épouvantail qu'il dresse pour protéger son jardin secret. Martin Eden est un monstre imbu de son moi, qui est allé trop loin dans son exaltation de la beauté idéale. La voix de la raison, celle d'un autre personnage du roman, le poète Brissenden, d'ailleurs beaucoup plus critique que Martin sur le monde de l'édition et de la bourgeoisie, était probablement la voix plus réfléchie du socialiste London. Ou, dernière hypothèse, c'est par l'évocation de différents personnages que London exposera et tentera de résoudre les contradictions et tensions psychiques entre ses désirs de gloire, de reconnaissance sociale et ses convictions socialistes lui venant de sa compassion pour le peuple de l'abîme.

Le roman de Jack London peut-il être révélateur de l'idéologie de l'American dream durant la période triomphale de l'Amérique industrielle au tournant du $20^{\circ}$ siècle 9 ? Pour répondre, quelques descriptions du contexte social dans lequel s'élabore cette quête du bonheur du héros serviront ici à montrer la participation de ce récit à la construction d'une idéologie faisant l'apologie du rêve américain. L'itinéraire social de Martin Eden suit parfois celui de London, parfois s'en écarte, dans le jeu de la fiction, mais aussi dans une recherche de cohérence et de sens dans la production d'un récit de vie qui soit réaliste tout en réussissant à susciter l'envie, le rêve, la compassion pour un homme et son destin.

D'où vient Martin Eden? Martin sort de nulle part, il débarque à San Francisco. II a un passé de matelot; il a connu la prison, les bagarres, les femmes des ports et des îles. II n'a pas vraiment de famille : ses parents sont morts et ses frères et sœurs, dispersés. II se débrouille seul depuis l'âge de 11 ans. London décrit donc l'enfance d'un orphelin venu du monde ouvrier. Dans ce roman, comme dans les différents récits autobiographiques de London, les jeunes ambitieux sont irrémédiablement seuls et se forgent un nom à partir de leurs seules réalisations. Le fantasme de l'auto-création peut se relier chez Jack London à son illégitimité. Bien qu'élevé par l'époux de sa mère, John London, et portant son nom, Jack London sait que sa 
mère a tenté de se suicider lors de sa grossesse et que son géniteur, Henry Chaney l'a quittée avant sa naissance en refusant de reconnaître son enfant. Fils de personne, il se produit une identité idéale à la mesure de son humiliation de n'avoir pas été voulu et reconnu par ses parents. L'écriture de récits à haute teneur biographique lui permet ainsi de jouer à ce jeu de recréation généalogique et de marquer d'une réussite encore plus éclatante ses réalisations personnelles.

\section{L'ascension sociale}

Martin Eden pénètre dans le monde de la bourgeoisie à l'aide de ses poings. Dans le port, il aide un jeune homme à se tirer d'une rixe avec des ivrognes. Arthur Morse invite le marin chez lui. Les premières pages du roman racontent ce premier contact de Martin avec la grande bourgeoisie d'affaires et le véritable choc culturel et émotionnel qui s'ensuivra. Choc culturel qui se manifestera quand Martin réalisera qu'il a honte pour la première fois de sa vie, honte de la piètre image qu'il donne ainsi de lui. Gauche, il louvoie comme sur un bateau entre le piano et la table centrale, tâchant d'éviter les embûches. Ses mots, sa tenue, tout dit qu'il vient d'un autre monde, celui de la mer. II ne sait pas où mettre les mains, tente d'imiter les gestes des autres et se croit dans un livre. II rage intérieurement parce que son manque d'assurance peut passer pour de l'ignorance et il tâche de ne pas être trop ridicule. Choc émotionnel car il fait la connaissance de la sœur d'Arthur, Ruth, une divine apparition. La bourgeoise a déjà classé socialement Martin, en passant en revue ses habits bon marché, mais la femme a aussi remarqué sa peau bronzée et ses biceps. Dès le choc de cette intrusion dans le monde de la richesse et du raffinement intellectuel, comme stimulé par le regard de cette femme ironique, certes, mais pas insensible, Martin se donne pour but de la conquérir : "vivre pour elle... se battre et mourir pour elle ${ }^{10}$ ». Puis, réalisant ce que cette conquête lui coûtera d'efforts, lui l'ignorant qu'il découvre être auprès de la jeune fille, il lui lance comme une menace, un défi : "Je ne connais pas grand-chose à tout ça. C'est pas dans mes cordes. Mais je serai bientôt à la hauteur ${ }^{11}$ ".

Tout le roman tourne autour de la longue ascension que cela suppose, tant la distance sociale est grande entre eux. Cette montée s'accompagne de son désenchantement progressif à mesure qu'il y accède et en découvre les limites intellectuelles et morales. Pour parvenir à ses fins, Martin commencera par fréquenter la bibliothèque 
publique, où il croit pouvoir s'instruire sur le savoir-vivre, clé apparente du savoir. Martin arpente d'abord le rayon philosophie et se surprend qu'elle pût susciter autant d'écrits. Comment dominer tout cela? "Le pouvoir était ici, dans les livres ${ }^{12}$ ». Les subtilités de la langue qu'il faut acquérir, les manières de table à maîtriser sont les tickets d'entrée du monde des idées. Sa boulimie de lecture l'entraîne donc dans une sorte de révolution hygiénique et morale : il se lave tous les matins, repasse son pantalon, arrête de boire et ne répond plus aux avances des filles dans la rue. II a même honte de ces avances; chacune le rabaisse à ses origines, à sa misère, à sa brutalité; il se sent démasqué. L'image de l'une de ces jeunes filles rencontrées un soir, Lizzie Connolly, servira tout au long du récit comme illustration du côté animal de la femme, celui de l'amour charnel, absolu, comme celui des femmes qu'il a connu jadis. La honte des origines, l'animalité du désir se retrouvent d'ailleurs dès le début et tout au long de ce récit; elles sont le sentiment et la pulsion qui nourrissent sa soif de reconnaissance sociale.

Martin veut écrire, devenir un homme de lettres, un géant de ce monde. Il écrit ses aventures de voyages, des chasses au trésor, à la baleine, envoie le tout aux journaux et attend qu'on le publie en lui payant chaque mot qu'il s'enflamme d'aligner dans des feuilletons de plus en plus nombreux, et non publiés. II travaille fort, et éprouve une sorte de fièvre, celle du bonheur de la création.

II était profondément heureux. La vie volait haut. Sa fièvre ne retombait jamais. Le bonheur de créer, qui était censé n'appartenir qu'aux dieux, était en lui. Et en lui était la vraie vie; tout le reste, les odeurs de lessive et de légumes pourris, (...) n'était qu'un rêve. Le monde réel était dans son esprit et les histoires qu'il écrivait en étaient l'expression ${ }^{13}$.

Curieusement, ce sont des journées de dix-neuf heures de travail qu'il décrit et c'est à la force de sa volonté et de son endurance qu'il parvient à enrichir ses connaissances. Ici, les recettes du bonheur à l'américaine restent donc valables. Labeur, mépris des besoins primaires, volonté et bonne étoile ou sentiment d'être différent des autres, de ceux de son monde, ceux que la société traite comme des bêtes de somme.

Puis, il a une révélation, au détour d'un parc. Un clochard harangue la foule en exposant les idées évolutionnistes de Spencer et c'est la découverte d'un monde où tout est relié. Tout s'explique. «ll 
n'y avait pas de hasards. II n'y avait que des lois ${ }^{14}$ ». Enfin un penseur qui lui présente "un modèle d'univers aussi parfait et soigné qu'une maquette de bateau dans une bouteille de verre ${ }^{15}$ ", contrairement à Kant qui, dit-il, lui avait fait douter de ses facultés intellectuelles! Ces nouvelles connaissances, Martin va vouloir les utiliser pour enrichir son écriture. Pour lui, la culture doit être un tremplin vers le succès.

Jack London est un self-made man. Dans Martin Eden, nulle trace trop visible de son passé de vagabond et de sa participation à la ruée vers l'or, mais il évoque certains épisodes de sa vie d'ouvrier : la blanchisserie, les amourettes avec les filles de la rue, les bagarres, la vie de misère de sa sœur, mariée à un épicier. Dans ces récits, la résistance physique à l'exploitation se heurte à la violence des rapports de domination. Femmes humiliées ou méprisées, abrutissement dans l'alcool comme façon de tenir le coup, bagarres pour conquérir les femmes, voilà quelques-unes des images illustrant cette loi du plus fort qui règne sans partage dans la culture de la pauvreté. La descente aux enfers du monde ouvrier dont il vient et dont il espérait sortir le convainc qu'à tout prendre la liberté est davantage dans le travail de l'écriture ou dans l'errance du hobo. Là au moins on ne tue pas le rêve. Jack London avait sillonné à pied les routes américaines vers Washington en 1894. II faisait partie d'un vaste groupe de chômeurs réclamant un programme de création d'emplois. Le récit de cette épopée de la faim, il la racontera dans Les Vagabonds du raill6 où comme dans Martin Eden, il prend soin d'évacuer les éléments trop sordides de la vie d'errance, comme s'il reconnaissait que ces hommes restaient en quête de liberté, des loups qui ne développaient aucune solidarité humaine, à la différence des ouvriers qui se laissaient réduire à être des bêtes de somme.

Jack London croit-il au rêve américain, à savoir que chacun peut faire son bonheur? L'écriture et l'apprentissage du métier se font de façon quasi miraculeuse chez Martin Eden. II apprend à maîtriser la dactylographie en un après-midi; il rédige à la chaîne nouvelles, essais, romans, pendant plusieurs heures par jour. Véritable forçat de l'industrie littéraire, Martin Eden serait le prototype de l'écrivain prolétaire, s'il existait. Cette austérité de la vie de l'écrivain correspond à celle de London à ses débuts, lui qui a écrit de façon si prolixe : nulle trace ici de panne d'inspiration ou de personnages rétifs à suivre l'aventure de l'écriture. Jack London a lui-même écrit plus de cinquante volumes en moins de vingt ans! Nul romantisme donc, mais un travail acharné, dans des conditions misérables, et le tout dirigé 
dans un seul but : produire un texte qui pourra être retenu par les revues de l'Est américain et qui pourra être payé, tant le mot. Ces revues publient des feuilletons, sur des sujets où la morale conventionnelle rejoint les thèmes de la littérature populaire. Les écrivains de l'époque, Mark Twain (voir son Autobiographie ${ }^{17}$ dont plus du tiers est consacrée à ses déboires avec les éditeurs) en est le prototype réussi, devaient passer par cette voie pour être publiés, ce qui supposait une bonne connaissance des recettes et clichés de cette littérature. L'auteur n'a d'ailleurs aucun regard critique sur les écrits de Martin Eden et même si le succès devait lui apprendre plus tard à négocier avec les éditeurs et à complaire au goût du public, il ne remet pas en question les certitudes de son personnage : il a du talent, et puisqu'il écrit à longueur de journée, cet apprentissage devrait le mener au succès ${ }^{18}$. La croyance au mérite individuel et à la légitimité du succès pour les individus de talent sont au cœur de cette idéologie du succès qui marque la fin du $19^{\mathrm{e}}$ siècle. Pensons à Carnegie, Rockefeller, et à tant d'autres qui doivent leur succès à leur capacité à le légitimer socialement et aux yeux de leurs adversaires. Cela passe par la fabrication d'un récit de vie faisant du travail l'instrument principal de la richesse et par la philanthropie ostentatoire, comme justification morale du droit au profit. Les "millionnaires» de la fin du $19^{\mathrm{e}}$ siècle inspirent le respect et l'émulation qui seront à la base de cette croyance solide au rêve américain. Si la fortune effraie par ses tendances à la concentration, c'est qu'elle s'oppose à cette autre idée-force de l'époque, celle de la liberté d'entreprendre : chacun a le droit de se lancer dans les affaires et seul le trust apparaît comme l'ennemi du bien public ${ }^{19}$. L'image de l'entrepreneur est donc plutôt positive et elle suscite plus souvent l'admiration que la critique 20 . Les premiers critiques socialistes font plus le procès du darwinisme social et des effets de la société industrielle sur les masses exploitées que celui des méthodes d'enrichissement des capitalistes. Selon eux, l'Amérique reste toujours la terre des opportunités, l'éden qui a su échapper aux horreurs de la société de classes européennes que sont les privilèges et les inégalités. La vie serait un combat plus juste dans cette nouvelle contrée; y réussiraient ceux qui se battent ou qui ont l'avantage de la bonne naissance, de la bonne race, de la bonne hérédité. Autant de privilèges naturels dont jouiraient tous les Américains blancs, descendants des fiers pionniers de l'indépendance. Le darwinisme ou plus spécialement son interprétation par Spencer devait se mêler aux autres éléments de la 
croyance très américaine en son exceptionnel destin dans le concert des nations pour solidifier cette croyance au progrès, à l'égalité des chances et à l'avènement de l'abondance.

Cette étape de la légitimation et de la justification de soi aux yeux des autres, Martin Eden ne parviendra pas à la franchir. Sa mort volontaire est donc un désaveu de lui-même. On peut examiner ici le rôle de la honte, ce sentiment minant l'estime de soi et sabotant toute réussite personnelle comme hypothèse pour expliquer pourquoi London a finalement donné une fin tragique à Martin Eden.

\section{La honte, aiguillon dans la quête du bonheur}

Dans chacune des rencontres entre Martin et la famille Morse le désir de Martin d'être aimé par Ruth le conduit chaque fois à se nier lui-même, à imiter les autres et à manifester sa volonté de s'améliorer pour obtenir son estime. La honte qu'il éprouve lors de ces rencontres a un effet de contrôle social sur lui, contrôle d'abord physique : ses manières, son langage, et contrôle moral : l'interdit d'amour et de réciprocité de sentiments de la jeune fille. Contrôle social enfin, puisque ses ambitions littéraires ne sont pas reconnues. Seuls les universitaires peuvent légitimement se consacrer entièrement à l'écriture. Ils ont les études, mais surtout le temps et l'argent nécessaires à ce loisir. On rappelle constamment à Martin qu'il doit chercher un travail, Ruth, sa sœur, son mari, et c'est chaque fois une gifle qu'on lui sert, un déni de son être et de sa créativité. Dans la plupart des sociétés, la condition minimale de la liberté est celle de s'appartenir. Dans la société capitaliste, l'esclave prolétaire n'est pas libre de son temps et des produits de sa créativité ou de son travail. Le contrôle social et ses variantes morales et corporelles servent justement à rappeler à chacun sa place et ses privilèges. La honte apparaît ainsi comme un sentiment social qui concerne l'identité du sujet pris dans la contradiction entre son désir d'être et le regard que porte autrui sur lui21. Comme Martin souhaite intégrer cette classe, il doit aussi intérioriser cette honte de soi qui lui est signifiée. Tout au long du roman, et souvent après une visite dans l'autre monde, Martin se regarde dans le miroir et cherche à deviner si le nouvel homme, l'écrivain, le savant pointe sous le voyou. Au début, il ne voit que le marin, et s'il sait reconnaître ses mérites : des yeux francs, des lèvres sensuelles de combattant et d'amant, des dents solides, il se déprécie en se comparant à Ruth. Elle, elle a les dents propres de celles qui se les lavaient tous les jours et les mains douces, de celles qui n'ont 
jamais eu à travailler22. Puis à mesure qu'il s'instruit, qu'il gagne de l'assurance dans son projet de devenir écrivain, le visage dans le miroir change. S'il a encore des doutes sur les folles prétentions qui sont les siennes et qu'il a le réflexe de se remettre à sa place, ses ambitions prennent le dessus. En se regardant dans la glace, de retour d'une soirée avec Ruth, il voit encore mal celui qu'il veut devenir. "Qui es-tu? Où est ta place?23» Est-il ce travailleur vulgaire vivant dans la sueur et la puanteur? Sa place est-elle plutôt parmi les "gens de son rang"? II cherche dans son regard ce qui lui permet d'oser s'arracher de la glèbe et "aimer une femme-esprit qui le dépasse24». Progressivement, l'écriture a fait évoluer son image de soi, mais il balance encore entre la honte des origines et l'exaltation démesurée de son idéal de réussite. Plus loin, l'image se dédouble. II voit apparaître un voyou, crâneur, au chapeau à bord plat. $\mathrm{Ce}$ fantôme de sa jeunesse lui rappelle qui il était hier, pas mieux que ceux qu'il méprisait aujourd'hui. Le nouveau Martin, sobre, sage, studieux sourit à cet inconnu qu'il n'est plus. II acquiert le respect du nouvel homme qu'il est devenu; son ego est devenu immense, démesuré; il se croit au-dessus de tous, seul. II cèdera même à la tentation de se croire tout-puissant en faisant le bonheur de tous les humiliés qui ont croisé son chemin et qui méritaient de prendre une revanche sociale : sa sœur, sa logeuse, Dawson, le blanchisseur, Lizzie. Mais la vengeance est un plat qui ne procure aucune chaleur: c'est de la fatigue qu'il éprouve après avoir refusé de renouer avec Ruth, pas de la jouissance. Aucun regard ne peut plus confirmer sa valeur et son humanité, pas même le sien. II ne se voit plus et il refuse le regard des autres. C'est ici que les idées du surhomme de Nietzsche servent de support au sentiment d'orgueil si fréquent dans les récits de ceux qui ont voulu traverser la barrière des classes. Selon Gauléjac qui a étudié les névroses de classe et les effets psychosociaux des trajectoires sociales ascendantes, «l'orgueil, c'est le narcissisme exacerbé, l'obsession de soi-même" de celui qui «tente de sortir de sa dépendance au regard de l'autre et qui ne fait que s'y soumettre 25 .

La fin du roman montre un homme déchiré entre deux mondes, parce que, s'il a voulu échapper à la misère de l'un, il n'a pu intégrer ou rejeter complètement le regard de la bourgeoisie sur son passé.

\section{Martin Eden et le rêve socialiste}

Martin Eden fait la connaissance d'un poète tuberculeux qui le 
guidera dans les derniers jours de sa vie. Véritable ange annonciateur, Brissenden, probablement l'alter ego de London dans ce récit s'exclame : "Ce n'est pas le succès qui vous procurera le bonheur, mais l'écriture. Je sais de quoi je parle. Vous le savez aussi. La beauté vous fait mal, c'est une souffrance éternelle, une plaie qui ne guérit pas, une lame de feu. Pourquoi courtiser les revues? Que la beauté soit votre seule fin! (...) Laissez là la gloire et la fortune, embarquez-vous demain sur un bateau et reprenez la mer26». II l'enjoint à laisser tomber les filles de la bourgeoisie et à aimer une femme "délurée et enflammée, qui rit de la vie, se moque de la mort et sait aimer27".

Brissenden l'amène à une réunion chez les socialistes avec la conviction que le salut de Martin se trouve dans l'abandon de sa croyance nietzschéenne dans l'arrivée d'un valeureux cavalier, venant balayer les hordes d'esclaves. II croit sincèrement que le socialisme pourrait le sauver de la désillusion. Martin y assiste et y fait part de sa croyance dans la loi biologique du développement et de sa nécessité pour accroître la force des États-Unis. On y retrouve pêle-mêle les arguments nietzschéens concernant la déchéance inévitable des sociétés fondées sur l'esclavage volontaire, mais aussi la dénonciation socialiste de la société américaine où même les enfants triment au service de l'oligarchie marchande. II termine sa harangue en défiant les socialistes de nommer la loi par laquelle ils souhaitent remplacer la terrible loi du plus fort, s'ils souhaitent conserver le pouvoir28.

Jack London n'était pas un socialiste des plus fidèles à la ligne du parti. Plusieurs témoignages et ses propres écrits rappellent toutefois qu'il a toujours dénoncé la douce soumission des pauvres dans les sociétés capitalistes. Pensons par exemple à The People of the Abyss, décrivant la vie des clochards, voleurs et prostituées d'un quartier de Londres. Le socialiste américain London ne pourra que constater les différences entre leur apathie et le rêve de promotion sociale de l'aristocratie ouvrière des villes américaines. Les intellectuels socialistes américains du début du $20^{\circ}$ siècle étaient aussi fascinés par les idées scientistes de Spencer, Darwin, et même par la pensée eugéniste de Haeckel dont on retrouve plusieurs traces dans l'œuvre de London ${ }^{29}$. Chez lui, le darwinisme se résumait en une croyance à la loi de la survie du plus fort, du plus adapté, ce qui faisait de ces êtres sauvages des loups pour les petits esprits de la bourgeoisie ou les victimes de la classe ouvrière. En fait, London ne 
voyait pas de différence entre les idées évolutionnistes de Spencer et les théories de Nietzsche. La théorie de l'évolution recouvrirait l'idée de l'évolution du moi de Nietzsche, de même que la volonté de puissance serait un autre concept pour illustrer la loi de la survie de l'espèce ${ }^{30}$. Cet amalgame entre des pensées bien différentes fait partie des contradictions et tensions des écrits de Jack London qui se révèlent surtout ceux d'un autodidacte et romancier.

\section{Le prix de la gloire : la solitude}

La visite de Martin Eden dans l'antre du socialisme sera rapportée dans les journaux; ses idées seront travesties au point qu'on en fait le leader des socialistes d'Oakland. Cette publicité provoquera la frayeur de Ruth qui lui envoie une lettre de rupture. Martin sent que quelque chose s'est cassé, "une page était tournée» et il se résout à terminer son dernier livre, dont le titre est révélateur : Trop tard, puis à attendre la suite des événements. Le succès frappe enfin à la porte. L'argent rentre mais l'amour est mort. II s'explique mal le revirement des magazines qui acceptent aujourd'hui ce qu'ils avaient refusé pendant deux ans. "Une bizarrerie du destin», conclut-il. II explique qu'il repousse les marques de reconnaissance sociale parce qu'il voulait être aimé pour lui-même, pour ses œuvres et non pour avoir son nom dans la liste des invités d'une réception chic.

C'est alors qu'un autre rêve va naître. Cet espace de bonheur, ce nouvel éden se trouve dans les îles: Tahiti, Tuamotu et les Marquises. Les filles enguirlandées de fleurs et les mers du Sud l'appellent et il se voit voguant à leur rencontre. II rêve à la beauté des îles, un monde d'aventures où il pourra oublier l'autre monde et ses illusions. Il décide de ne plus écrire. C'est là le plus grand renoncement de Martin Eden, lui qui avait découvert que la réelle beauté du monde est celle qu'il avait mise dans ses récits. Cette errance maritime et imaginaire, c'est l'occasion de refaire sa vie et peut-être de reconstruire le rêve.

Avant de tout quitter, il se laisse dériver dans l'univers social qu'il a connu, la paurreté et dans celui auquel il aspirait, le beau monde. II se sent au-dessus de la mêlée, ailleurs. II fait des adieux, des deuils de soi-même, dirons-nous, de ces illusions qui ont animé sa quête. S'il n'a plus foi en ce monde qu'il rejette, il cherche à réaliser le rêve de tous les pauvres hères qu'il a rencontrés. À chacun de ces personnages, Martin apportera son soutien financier pour réaliser un souhait. Ces rêves sont quelques-unes des réalisations de London : achat 
d'un ranch, d'une entreprise qui n'exploite pas ses ouvriers, emploi de personnel domestique pour son épouse. Enfin, il fait ses adieux à l'amour. À Lizzie Connolly, cette jeune femme qui lui avait souri un soir, il ne peut rien offrir, que de l'argent, "chose superflue dont il peut se départir sans souffrir, tandis qu'elle s'offrait elle-même, avec ses disgrâces, ses turpitudes, ses péchés et toutes ses espérances ${ }^{31}$ ». II finira par la convaincre de suivre des cours du soir et par la traiter comme une sœur. II dit qu'il est trop différent d'elle, mais quand la glotte de la jeune femme se serre d'avoir été repoussée comme amoureuse, n'est-ce pas sa propre dignité qu'il reconnaît chez elle? Le sentiment de honte chez les autres, il le devine, même s'il ne veut plus le partager. N'est-ce pas sa propre humanité que refuse Martin Eden? Sa dernière rencontre avec Ruth, venue s'offrir à lui dans sa chambre d'hôtel met le point final à la série de ses désillusions. II sait qu'elle veut maintenant de lui comme époux parce qu'il est célèbre et riche, et il lui avoue qu'il n'a plus foi en rien et n'éprouve plus aucun désir pour elle.

Il embarque sur la Mariposa en route vers Tahiti et réalise qu'il n'a plus de place dans ce monde. II a refusé la gloire, l'amour, le bonheur. II en a vu le caractère éphémère et factice. II se sent écartelé entre tous ces univers sociaux auxquels il refuse de s'identifier :

Il était en perdition. Dans les hautes sphères, personne ne s'intéressait à Martin Eden pour lui-même et il ne pouvait pas redescendre dans les bas-fonds où on l'avait aimé. II ne voulait plus d'eux. II ne supportait pas davantage les gars de la marine que les frivoles passagers de première classe et les jeunes fêtards du pont supérieur ${ }^{32}$.

L'éden, le paradis est perdu : quand matelot, il voyait tous ces passagers se prélasser au soleil, «il croyait voir des princes en paradis. Et à présent qu'il était des leurs, qu'il était le grand homme du bord, le roi de la fête, assis à la droite du capitaine, il retournait en vain vers le poste d'équipage et la chaufferie en quête d'un paradis perdu : il n'avait jamais découvert celui qu'il cherchait et ne retrouvait plus celui qu'il avait cherché33". Y a-t-il un nom pour ce sentiment d'écartèlement social?

La vie l'aveugle, lui fait mal. II renonce à poursuivre l'œuvre de restauration du rêve dans les îles, tant cette vie l'accable. II trouve la réponse chez le poète anglais Swinburne. La délivrance était proche. "La mort était là pour endormir la douleur dans un sommeil éternel34". 
Se hissant par le hublot, il se laisse engloutir par l'océan.

La mort est la fin ultime. La fin du roman. La fin du récit. La fin de la quête du bonheur. La fin de la souffrance. On entend l'écho de ces vers de Longfellow qui avaient hanté Martin Eden :

La mer est calme et profonde.

Toute chose dort sous l'onde.

Un seul pas et s'en est fait,

Un plongeon, une bulle et plus rien 35 .

Le gouffre qui sépare les classes et que la bourgeoisie s'ingénie à rendre le plus infranchissable possible en maintenant solides les barrières de la morale et du bon goût, Martin Eden a cru pouvoir le combler en devenant écrivain, en captivant ses lecteurs par le récit pittoresque d'aventures d'êtres hors du commun. Jack London, l'écrivain des grands espaces, des récits authentiques d'aventures dans les îles et dans les contrées rudes du Nord, a lui aussi mis en vedette des êtres plus vivants que nature, inspirés d'une énergie vitale telle que plusieurs personnages deviennent des animaux féroces mais nobles, comme Croc Blanc. Jack London fait de Martin un homme-chien devenu loup, solitaire et sauvage, qui ne peut trouver le repos ni dans le ciel avec les aigles, ni dans les îles avec ses rêves, mais au fond des ténèbres de l'océan, seul lieu où peuvent se réfugier les monstres, ceux qui ne croient plus à leur humanité. Le récit de la mort de Martin Eden peut probablement être considéré comme une façon d'apprivoiser la mort d'une partie de soi par Jack London, celle du rêve de produire une vie unique, à soi, malgré les contingences sociales et personnelles qui la condamnent à être aussi celle d'un autre, un inconnu, celle de Martin Eden par exemple.

Le récit de Martin Eden a servi ici d'itinéraire possible dans la quête du succès littéraire comme voie d'accès au bonheur d'un Américain du début du $20^{\circ}$ siècle. Par la richesse de sa description sociale des milieux sociaux californiens, tant celui des affaires que celui des intellectuels progressistes, Jack London reprend plusieurs événements de sa propre vie pour dresser un constat d'échec d'une vie tout entière tournée vers l'idéal d'une reconnaissance sociale qui reposerait sur le seul mérite individuel ou le génie. La solitude et le désenchantement apparaissent ici comme les rançons d'un tel succès.

L'examen des rapports entre le roman et la vie de Jack London a aussi révélé que si au début Martin Eden se reflétait dans le miroir de 
Jack London, peu à peu le récit a créé un personnage dans lequel Jack London n'a pas voulu se reconnaître. Le suicide de Martin Eden s'interprète ainsi comme une façon de briser la surface de l'eau et l'image du visage de l'autre qu'on cherche à brouiller quand on s'y coule. En reprenant l'idée de Ricœur que l'on a soulevée au début, on avancera même que London n'acceptait pas qu'on reconnaisse ce récit comme le sien. Martin Eden illustre finalement la visée éthique au cœur de toute entreprise de livrer sa vie au regard d'autrui et la difficulté de London de s'en tenir à une définition purement égoïste du bonheur. Avec ce roman, l'Amérique du début du 20e siècle, traversée par l'idéologie du progrès et de l'individualisme, permettait aussi l'émergence d'un rêve humaniste et socialiste de libération de l'aliénation sociale.

\section{Michèle Emond Socioologie, Collège Édouard-Montpetit}

1. Paul Ricœur, «La souffrance n'est pas la douleur», Autrement, n 142, février 1994, p. 63.

2. René Denis, Message sur le bonheur, courriel à l'auteure, 4 août 2003.

3. Jack London, Martin Eden, Paris, Phébus, 2001.

4. C'est ce portrait qui se dégage surtout des premières biographies de London. Voir Iving Stone. Sailor on Horseback : The Biography of Jack London, 1938; Philip S. Foner, Jack London : American Rebel, 1947.

5. L'analyse des multiples facettes de la construction sociale de la biographie de Jack London a été faite par Clarice Stasz, "The social construction of biography : The Case of Jack London", Modern Fiction Studies (Spring, 1976), 51-71. Voir aussi Clarice Stasz, Biographies of Jack London, adresse url : sunsite.berkeley.edu/londonbio.html.

6. Francis Lacassin, “Jack London n'était pas Martin Eden mais il le devint», Préface à Martin Eden, Paris Hachette, 1984.

7. Paul Ricœur, "Sixième étude. Le soi et l'identité narrative", Soi-même comme un autre, Paris, Seuil, 1990, p. 193.

8. Paul Ricœur, "Cinquième étude. L'identité personnelle et l'identité narrative", op. cit.

9. André Kaspi, Les Américains. I. Naissance et essor des États-Unis 1607-1945, Paris, Seuil, coll. Points, 1986, chap. 7 "Le triomphe de l'Amérique industrialiste».

10. Martin Eden, p. 22.

11. Ibid., p. 25.

12. Ibid., p. 58.

13. Ibid., p. 109.

14. Ibid., p. 124. 
15. Ibid.

16. Francis Lacassin, "Jack London ou Les crocs rouges de Croc-Blanc", Préface. Jack London. Romans, récits et nouvelles du Grand Nord, Paris, Robert Laffont, 1983, p. 5.

17. Mark Twain, Autobiographie, Paris, Éditions du Rocher, 2003, $573 \mathrm{p}$.

18. Andrew Sinclair, Jack London, Paris, Pierre Belfond, 1979, p. 53.

19. André Kaspi, op. cit., p. 220.

20. Ibid., p. 218.

21. Vincent de Gaulejac, Les sources de la honte, Paris, Desclée de Brouwer, 1996, p. 302.

22. Martin Eden, p. 49-50.

23. Ibid., p. 121.

24. Ibid.

25. Vincent de Gaulejac, op. cit., p. 249.

26. Martin Eden, p. 306.

27. Ibid., p. 308.

28. Grande question à laquelle les États du $20^{\mathrm{e}}$ siècle ont apporté des réponses aussi terrifiantes que variées.

29. Joseph Sciambra, "The Philosophy of Jack London", adresse url : http: //sunsite.berkeley.edu/London/Essays/philosophy.html

30. Andrew Sinclair, Jack London, Paris, Belfond, 1977, p. 114.

31. Martin Eden, p. 388.

32. Ibid., p. 433.

33. Ibid., p. 433.

34. Ibid., p. 435.

35. Ibid., p. 290. 